\title{
Wijsman quasi-invariant convergence
}

\author{
ESRA GÜLLE and UĞUR ULUSU
}

ABSTRACT. In this study, we defined concepts of Wijsman quasi-invariant convergence, Wijsman quasistrongly invariant convergence and Wijsman quasi-strongly $q$-invariant convergence. Also, we give the concept of Wijsman quasi-invariant statistically convergence. Then, we study relationships among these concepts. Furthermore, we investigate relationship between these concepts and some convergence types given earlier for sequences of sets, too.

\section{INTRODUCTION AND BACKGROUND}

The concept of statistical convergence was firstly introduced by Fast [5] and this concept has been studied by Šalát [15], Fridy [6], Connor [4] and many others, too.

A sequence $x=\left(x_{k}\right)$ is statistically convergent to $L$ if for every $\varepsilon>0$

$$
\lim _{n \rightarrow \infty} \frac{1}{n}\left|\left\{k \leq n:\left|x_{k}-L\right| \geq \varepsilon\right\}\right|=0,
$$

where the vertical bars indicate the number of elements in the enclosed set.

Several authors have studied on the concepts of invariant mean and invariant convergent sequences (see, $[7,9,10,14,16,19]$ ).

Let $\sigma$ be a mapping of the positive integers into themselves. A continuous linear functional $\phi$ on $\ell_{\infty}$, the space of real bounded sequences, is said to be an invariant mean or a $\sigma$-mean if it satisfies following conditions:

(1) $\phi(x) \geq 0$, when the sequence $\left(x_{n}\right)$ has $x_{n} \geq 0$ for all $n$,

(2) $\phi(e)=1$, where $e=(1,1,1, \ldots)$, and

(3) $\phi\left(x_{\sigma(n)}\right)=\phi\left(x_{n}\right)$ for all $x \in \ell_{\infty}$.

The mappings $\sigma$ are assumed to be one-to-one and such that $\sigma^{m}(n) \neq n$ for all positive integers $n$ and $m$, where $\sigma^{m}(n)$ denotes the $m$ th iterate of the mapping $\sigma$ at $n$. Thus, $\phi$ extends the limit functional on $c$, the space of convergent sequences, in the sense that $\phi(x)=\lim x$ for all $x \in c$. limit.

In the case $\sigma$ is translation mappings $\sigma(n)=n+1$, the $\sigma$-mean is often called a Banach

It can be shown that

$$
V_{\sigma}=\left\{x=\left(x_{n}\right) \in \ell_{\infty}: \lim _{m \rightarrow \infty} \frac{1}{m} \sum_{k=1}^{m} x_{\sigma^{k}(n)}=L, \text { uniformly in } n\right\} .
$$

The concept of strongly $\sigma$-convergence was introduced by Mursaleen [8].

Received: 18.12.2018. In revised form: 08.04.2019. Accepted: 15.04.2019

2010 Mathematics Subject Classification. 40A05, 40A35.

Key words and phrases. Invariant convergence, quasi-invariant convergence, quasi-invariant statistical convergence, sequences of sets, Wijsman convergence.

Corresponding author: Esra Gülle; egulle@aku.edu.tr 
A sequence $x=\left(x_{k}\right)$ is said to be strongly $\sigma$-convergent to $L$ if

$$
\lim _{m \rightarrow \infty} \frac{1}{m} \sum_{k=1}^{m}\left|x_{\sigma^{k}(n)}-L\right|=0,
$$

uniformly in $n$.

In [17], Savaş generalized the concept of strongly $\sigma$-convergence as below:

$$
\left[V_{\sigma}\right]_{p}=\left\{x=\left(x_{k}\right): \lim _{m \rightarrow \infty} \frac{1}{m} \sum_{k=1}^{m}\left|x_{\sigma^{k}(n)}-L\right|^{p}=0, \text { uniformly in } n\right\},
$$

where $0<p<\infty$.

The concept of $\sigma$-statistically convergent sequence was introduced by Savaş and Nuray [18] as follows:

A sequence $x=\left(x_{k}\right)$ is said to be $\sigma$-statistically convergent to $L$ if for every $\varepsilon>0$

$$
\lim _{m \rightarrow \infty} \frac{1}{m}\left|\left\{k \leq m:\left|x_{\sigma^{k}(n)}-L\right| \geq \varepsilon\right\}\right|=0,
$$

uniformly in $n$.

Let $X$ be any non-empty set and $\mathbb{N}$ be the set of natural numbers. The function $f: \mathbb{N} \rightarrow P(X)$ is defined by $f(k)=A_{k} \in P(X)$ for each $k \in \mathbb{N}$, where $P(X)$ is power set of $X$. The sequence $\left\{A_{k}\right\}=\left(A_{1}, A_{2}, \ldots\right)$, which is the range's elements of $f$, is said to be sequences of sets.

Let $(X, \rho)$ be a metric space. For any point $x \in X$ and any non-empty subset $A$ of $X$, the distance from $x$ to $A$ is defined by $d(x, A)=\inf _{a \in A} \rho(x, a)$.

Throughout the paper we take $(X, \rho)$ as a metric space and $A, A_{k}$ as any non-empty closed subsets of $X$.

There are different convergence notions for sequence of sets. One of them handled in this paper is the concept of Wijsman convergence (see, [1, 2, 3, 20, 21, 22]).

A sequence $\left\{A_{k}\right\}$ is said to be Wijsman convergent to $\mathrm{A}$ if for each $x \in X$,

$$
\lim _{k \rightarrow \infty} d\left(x, A_{k}\right)=d(x, A)
$$

and it is denoted by $A_{k} \stackrel{W}{\rightarrow} A$.

A sequence $\left\{A_{k}\right\}$ is said to be bounded if for each $x \in X$, there exists an $M>0$ such that $\left|d\left(x, A_{k}\right)\right|<M$ for all $k$, i.e., if $\sup _{k}\left\{d\left(x, A_{k}\right)\right\}<\infty$.

The set of all bounded sequences of sets is denoted by $L_{\infty}$.

Nuray and Rhoades [11] defined the concept of Wijsman statistical convergence for sequences of sets.

A sequence $\left\{A_{k}\right\}$ is Wijsman statistically convergent to $A$ if for each $x \in X$ and every $\varepsilon>0$

$$
\lim _{n \rightarrow \infty} \frac{1}{n}\left|\left\{k \leq n:\left|d\left(x, A_{k}\right)-d(x, A)\right| \geq \varepsilon\right\}\right|=0
$$

and it is denoted by $s t-\lim _{W} A_{k}=A$.

Using the invariant mean, the concepts of Wijsman invariant convergence $\left(W V_{\sigma}\right)$, Wijsman strongly invariant convergence $\left[W V_{\sigma}\right]$ and Wijsman invariant statistical convergence $\left(W S_{\sigma}\right)$ were also introduced by Pancaroğlu and Nuray [13].

A sequence $\left\{A_{k}\right\}$ is said to be Wijsman invariant convergent to $A$ if for each $x \in X$

$$
\lim _{n \rightarrow \infty} \frac{1}{n} \sum_{k=1}^{n} d\left(x, A_{\sigma^{k}(m)}\right)=d(x, A),
$$

uniformly in $m$ and it is denoted by $A_{k} \stackrel{W V_{F}}{\longrightarrow} A$. 
A sequence $\left\{A_{k}\right\}$ is said to be Wijsman strongly invariant convergent to $A$ if for each $x \in X$

$$
\lim _{n \rightarrow \infty} \frac{1}{n} \sum_{k=1}^{n}\left|d\left(x, A_{\sigma^{k}(m)}\right)-d(x, A)\right|=0,
$$

uniformly in $m$ and it is denoted by $A_{k} \stackrel{\left[W V_{\sigma}\right]}{\longrightarrow} A$.

A sequence $\left\{A_{k}\right\}$ is Wijsman invariant statistically convergent to $A$ if for each $x \in X$ and every $\varepsilon>0$

$$
\lim _{n \rightarrow \infty} \frac{1}{n}\left|\left\{k \leq n:\left|d\left(x, A_{\sigma^{k}(m)}\right)-d(x, A)\right| \geq \varepsilon\right\}\right|=0,
$$

uniformly in $m$ and it is denoted by $A_{k} \stackrel{\left(W S_{\sigma}\right)}{\longrightarrow} A$.

Nuray [12] studied concepts of quasi-invariant convergence and quasi-invariant statistical convergence for real sequences in a normed space.

\section{WIJSMAN QUASI-INVARIANT CONVERGENCE}

In this section, we defined concepts of Wijsman quasi-invariant convergence, Wijsman quasi-strongly invariant convergence and Wijsman quasi-strongly $q$-invariant convergence. Also, we give the concept of Wijsman quasi-invariant statistically convergence. Then, we study relationships among these concepts. Furthermore, we investigate relationship between these concepts and some convergences types given earlier for sequences of sets.

Definition 2.1. A sequence $\left\{A_{k}\right\}$ is Wijsman quasi-invariant convergent to $A$ if for each $x \in X$

$$
\lim _{p \rightarrow \infty}\left|\frac{1}{p} \sum_{k=0}^{p-1} d_{x}\left(A_{\sigma^{k}(n p)}\right)-d_{x}(A)\right|=0,
$$

uniformly in $n=1,2, \ldots$ where $d_{x}\left(A_{\sigma^{k}(n p)}\right)=d\left(x, A_{\sigma^{k}(n p)}\right)$ and $d_{x}(A)=d(x, A)$. In this case, we write $A_{k} \stackrel{W Q V_{\sigma}}{\longrightarrow} A$.

Theorem 2.1. If a sequence $\left\{A_{k}\right\}$ is Wijsman invariant convergent to $A$, then $\left\{A_{k}\right\}$ is Wijsman quasi-invariant convergent to $A$.

Proof. Suppose that the sequence $\left\{A_{k}\right\}$ is Wijsman invariant convergent to $A$. Then, for each $x \in X$ and every $\varepsilon>0$ there exists an integer $p_{0}>0$ such that for all $p>p_{0}$

$$
\left|\frac{1}{p} \sum_{k=0}^{p-1} d_{x}\left(A_{\sigma^{k}(m)}\right)-d_{x}(A)\right|<\varepsilon,
$$

for all $m$. If $m$ is taken as $m=n p$, then we get

$$
\left|\frac{1}{p} \sum_{k=0}^{p-1} d_{x}\left(A_{\sigma^{k}(n p)}\right)-d_{x}(A)\right|<\varepsilon
$$

for all $n$. Since $\varepsilon>0$ is an arbitrary, we have

$$
\lim _{p \rightarrow \infty}\left|\frac{1}{p} \sum_{k=0}^{p-1} d_{x}\left(A_{\sigma^{k}(n p)}\right)-d_{x}(A)\right|=0
$$

uniformly in $n$. Therefore, the sequence $\left\{A_{k}\right\}$ is Wijsman quasi-invariant convergent to A. 
Definition 2.2. A sequence $\left\{A_{k}\right\}$ is Wijsman quasi-invariant statistically convergent to $A$ if for each $x \in X$ and every $\varepsilon>0$

$$
\lim _{p \rightarrow \infty} \frac{1}{p}\left|\left\{k \leq p:\left|d_{x}\left(A_{\sigma^{k}(n p)}\right)-d_{x}(A)\right| \geq \varepsilon\right\}\right|=0
$$

uniformly in $n$. In this case, we write $A_{k} \stackrel{W Q S_{\sigma}}{\longrightarrow} A$.

The set of all Wijsman quasi-invariant statistically convergent sequences will be denoted by $\left(W Q S_{\sigma}\right)$.

Theorem 2.2. If a sequence $\left\{A_{k}\right\}$ is Wijsman invariant statistically convergent to $A$, then $\left\{A_{k}\right\}$ is Wijsman quasi-invariant statistically convergent to $A$.

Proof. Suppose that the sequence $\left\{A_{k}\right\}$ is Wijsman invariant statistically convergent to $A$. In this case, when $\delta>0$ is given, for each $x \in X$ and every $\varepsilon>0$ there exists an integer $p_{0}>0$ such that for all $p>p_{0}$

$$
\frac{1}{p}\left|\left\{k \leq p:\left|d_{x}\left(A_{\sigma^{k}(m)}\right)-d_{x}(A)\right| \geq \varepsilon\right\}\right|<\delta,
$$

for all $m$. If $m$ is taken as $m=n p$, then we get

$$
\frac{1}{p}\left|\left\{k \leq p:\left|d_{x}\left(A_{\sigma^{k}(n p)}\right)-d_{x}(A)\right| \geq \varepsilon\right\}\right|<\delta
$$

for all $n$. Since $\delta>0$ is an arbitrary, we have

$$
\lim _{p \rightarrow \infty} \frac{1}{p}\left|\left\{k \leq p:\left|d_{x}\left(A_{\sigma^{k}(n p)}\right)-d_{x}(A)\right| \geq \varepsilon\right\}\right|=0
$$

uniformly in $n$. Therefore, the sequence $A_{k}$ is Wijsman quasi-invariant satatistically convergent to $A$.

Definition 2.3. A sequence $\left\{A_{k}\right\}$ is Wijsman quasi-strongly invariant convergent to $A$ if for each $x \in X$

$$
\lim _{p \rightarrow \infty} \frac{1}{p} \sum_{k=0}^{p-1}\left|d_{x}\left(A_{\sigma^{k}(n p)}\right)-d_{x}(A)\right|=0,
$$

uniformly in $n$. In this case, we write $A_{k} \stackrel{\left[W Q V_{\sigma}\right]}{\longrightarrow} A$.

Definition 2.4. Let $0<q<\infty$. A sequence $\left\{A_{k}\right\}$ is Wijsman quasi-strongly $q$-invariant convergent to $A$ if for each $x \in X$

$$
\lim _{p \rightarrow \infty} \frac{1}{p} \sum_{k=0}^{p-1}\left|d_{x}\left(A_{\sigma^{k}(n p)}\right)-d_{x}(A)\right|^{q}=0,
$$

uniformly in $n$. In this case, we write $A_{k} \stackrel{\left[W Q V_{\sigma}\right]^{q}}{\longrightarrow} A$.

The set of all Wijsman quasi-strongly $q$-invariant convergence sequences will be denoted by $\left[W Q V_{\sigma}\right]^{q}$.

\section{Theorem 2.3.}

i) If a sequence $\left\{A_{k}\right\}$ is Wijsman quasi-strongly q-invariant convergent to $A$, then this sequence is Wijsman quasi-invariant statistically convergent to $A$.

ii) If a sequence $\left\{A_{k}\right\} \in L_{\infty}$ and Wijsman quasi-invariant statistically convergent to $A$, then this sequence is Wijsman quasi-strongly q-invariant convergent to $A$.

iii) $\left(W Q S_{\sigma}\right) \cap L_{\infty}=\left[W Q V_{\sigma}\right]^{q}$ 
Proof. i) Suppose that the sequence $\left\{A_{k}\right\}$ is Wijsman quasi-strongly $q$-invariant convergent to $A$. For each $x \in X$ and every $\varepsilon>0$, following inequality is provided:

$$
\sum_{k=0}^{p-1}\left|d_{x}\left(A_{\sigma^{k}(n p)}\right)-d_{x}(A)\right|^{q} \geq \varepsilon^{q}\left|\left\{k \leq p:\left|d_{x}\left(A_{\sigma^{k}(n p)}\right)-d_{x}(A)\right| \geq \varepsilon\right\}\right|,
$$

for all $n$. If the both side of the above inequality are multipled by $\frac{1}{p}$ and after that the limit is taken for $p \rightarrow \infty$, we get

$$
\lim _{p \rightarrow \infty} \frac{1}{p} \sum_{k=0}^{p-1}\left|d_{x}\left(A_{\sigma^{k}(n p)}\right)-d_{x}(A)\right|^{q} \geq \varepsilon^{q} \lim _{p \rightarrow \infty} \frac{1}{p}\left|\left\{k \leq p:\left|d_{x}\left(A_{\sigma^{k}(n p)}\right)-d_{x}(A)\right| \geq \varepsilon\right\}\right| .
$$

Since the sequence $\left\{A_{k}\right\}$ is Wijsman quasi-strongly $q$-invariant convergent to $A$, the left side of inequality (2.1) is equal to 0 . Hence, we have

$$
\lim _{p \rightarrow \infty} \frac{1}{p}\left|\left\{k \leq p:\left|d_{x}\left(A_{\sigma^{k}(n p)}\right)-d_{x}(A)\right| \geq \varepsilon\right\}\right|=0
$$

uniformly in $n$. So, the proof is completed.

ii) Suppose that the sequence $\left\{A_{k}\right\} \in L_{\infty}$ and Wijsman quasi-invariant statistically convergent to $A$. Since $\left\{A_{k}\right\}$ is bounded, there exists an $M>0$ such that for each $x \in X$

$$
\left|d_{x}\left(A_{\sigma^{k}(n p)}\right)-d_{x}(A)\right| \leq M
$$

Also, since $\left\{A_{k}\right\}$ is Wijsman quasi-invariant statistically convergent to $A$, for each $x \in X$ and every $\varepsilon>0$ there exists a number $N_{\varepsilon} \in \mathbb{N}$ such that for all $p>N_{\varepsilon}$

$$
\frac{1}{p}\left|\left\{k \leq p:\left|d_{x}\left(A_{\sigma^{k}(n p)}\right)-d_{x}(A)\right| \geq\left(\frac{\varepsilon}{2}\right)^{1 / q}\right\}\right|<\frac{\varepsilon}{2 M^{q}},
$$

for all $n$. Now, we take the set

$$
G_{p}=\left\{k \leq p:\left|d_{x}\left(A_{\sigma^{k}(n p)}\right)-d_{x}(A)\right| \geq\left(\frac{\varepsilon}{2}\right)^{1 / q}\right\} .
$$

Thus, for each $x \in X$ we get

$$
\begin{aligned}
& \frac{1}{p} \sum_{k=0}^{p-1}\left|d_{x}\left(A_{\sigma^{k}(n p)}\right)-d_{x}(A)\right|^{q}= \frac{1}{p}\left(\sum_{\substack{k \leq p \\
k \in G_{p}}}\left|d_{x}\left(A_{\sigma^{k}(n p)}\right)-d_{x}(A)\right|^{q}\right. \\
&\left.+\sum_{k \leq p}\left|d_{x}\left(A_{\sigma^{k}(n p)}\right)-d_{x}(A)\right|^{q}\right) \\
& k \notin G_{p} \\
&<\frac{1}{p} p \frac{\varepsilon}{2 M^{q}} M^{q}+\frac{1}{p} p \frac{\varepsilon}{2} \\
&=\frac{\varepsilon}{2}+\frac{\varepsilon}{2}=\varepsilon,
\end{aligned}
$$

for all $n$. So, the proof is completed.

iii) If $(i)$ and $(i i)$ are considered together, we handle $\left(W Q S_{\sigma}\right) \cap L_{\infty}=\left[W Q V_{\sigma}\right]^{q}$. 
Lemma 2.1. If for each $x \in X$ and every $\varepsilon>0$ there exists numbers $p_{0}$ and $n_{0}$ such that for all $p \geq p_{0}$ and $n \geq n_{0}$

$$
\frac{1}{p} \sum_{k=0}^{p-1}\left|d_{x}\left(A_{\sigma^{k}(n p)}\right)-d_{x}(A)\right|<\varepsilon,
$$

then the sequence $\left\{A_{k}\right\}$ is Wijsman quasi-strongly invariant convergent to $A$.

Proof. Let $\varepsilon>0$ be given. Because of the hypothesis, for each $x \in X$ we can choose numbers $p_{0}^{\prime}$ and $n_{0}$ such that

$$
\frac{1}{p} \sum_{k=0}^{p-1}\left|d_{x}\left(A_{\sigma^{k}(n p)}\right)-d_{x}(A)\right|<\frac{\varepsilon}{2},
$$

for all $p \geq p_{0}^{\prime}$ and $n \geq n_{0}$. It is enough to prove that there exists a number $p_{0}^{\prime \prime}$ such that

$$
\frac{1}{p} \sum_{k=0}^{p-1}\left|d_{x}\left(A_{\sigma^{k}(n p)}\right)-d_{x}(A)\right|<\varepsilon,
$$

for all $p \geq p_{0}^{\prime \prime}$ and $0 \leq n \leq n_{0}$. If $p_{0}$ is taken as $p_{0}=\max \left\{p_{0}^{\prime}, p_{0}^{\prime \prime}\right\}$, then the following inequality is hold:

$$
\frac{1}{p} \sum_{k=0}^{p-1}\left|d_{x}\left(A_{\sigma^{k}(n p)}\right)-d_{x}(A)\right|<\varepsilon,
$$

for all $p \geq p_{0}$ and $n$. The number $n_{0}$ is a constant due to the its selection. Thus, we can take as

$$
\sum_{k=0}^{n_{0}-1}\left|d_{x}\left(A_{\sigma^{k}(n p)}\right)-d_{x}(A)\right|=T .
$$

Now, when considering the inequality (2.2) for $0 \leq n \leq n_{0}$ and $p \geq n_{0}$, we get

$$
\begin{aligned}
\frac{1}{p} \sum_{k=0}^{p-1}\left|d_{x}\left(A_{\sigma^{k}(n p)}\right)-d_{x}(A)\right|= & \frac{1}{p} \sum_{k=0}^{n_{0}-1}\left|d_{x}\left(A_{\sigma^{k}(n p)}\right)-d_{x}(A)\right| \\
& +\frac{1}{p} \sum_{k=n_{0}}^{p-1}\left|d_{x}\left(A_{\sigma^{k}(n p)}\right)-d_{x}(A)\right| \\
= & \frac{T}{p}+\frac{1}{p} \sum_{k=n_{0}}^{p-1}\left|d_{x}\left(A_{\sigma^{k}(n p)}\right)-d_{x}(A)\right| \\
\leq & \frac{T}{p}+\frac{\varepsilon}{2} .
\end{aligned}
$$

If $p$ is taken sufficiently large, we can write

$$
\frac{T}{p}+\frac{\varepsilon}{2}<\varepsilon
$$

So, the sequence $\left\{A_{k}\right\}$ is Wijsman quasi-strongly invariant convergent to $A$.

Lemma 2.2. If for each $x \in X$ and every $\varepsilon, \delta>0$ there exists numbers $p_{0}$ and $n_{0}$ such that for all $p \geq p_{0}$ and $n \geq n_{0}$

$$
\frac{1}{p}\left|\left\{0 \leq k \leq p-1:\left|d_{x}\left(A_{\sigma^{k}(n p)}\right)-d_{x}(A)\right| \geq \varepsilon\right\}\right| \leq \delta,
$$

then the sequence $\left\{A_{k}\right\}$ is Wijsman quasi-invariant statistically convergent to $A$. 
Proof. Let $\varepsilon, \delta>0$ be given. Because of the hypothesis, for each $x \in X$ we can choose numbers $p_{0}^{\prime}$ and $n_{0}$ such that

$$
\frac{1}{p}\left|\left\{0 \leq k \leq p-1:\left|d_{x}\left(A_{\sigma^{k}(n p)}\right)-d_{x}(A)\right| \geq \varepsilon\right\}\right|<\frac{\delta}{2},
$$

for all $p \geq p_{0}^{\prime}$ ve $n \geq n_{0}$. It is enough to prove that there exists a number $p_{0}^{\prime \prime}$ such that

$$
\frac{1}{p}\left|\left\{0 \leq k \leq p-1:\left|d_{x}\left(A_{\sigma^{k}(n p)}\right)-d_{x}(A)\right| \geq \varepsilon\right\}\right|<\delta,
$$

for all $p \geq p_{0}^{\prime \prime}$ and $0 \leq n \leq n_{0}$. If $p_{0}$ is taken as $p_{0}=\max \left\{p_{0}^{\prime}, p_{0}^{\prime \prime}\right\}$, then the following inequality is hold:

$$
\frac{1}{p}\left|\left\{0 \leq k \leq p-1:\left|d_{x}\left(A_{\sigma^{k}(n p)}\right)-d_{x}(A)\right| \geq \varepsilon\right\}\right|<\delta,
$$

for all $p \geq p_{0}$ and $n$. The number $n_{0}$ is a constant due to the its selection. Thus, we can take as

$$
\left|\left\{0 \leq k \leq n_{0}-1:\left|d_{x}\left(A_{\sigma^{k}(n p)}\right)-d_{x}(A)\right| \geq \varepsilon\right\}\right|=H .
$$

Now, when considering the inequality (2.3) for $0 \leq n \leq n_{0}$ and $p \geq n_{0}$, we get

$$
\begin{aligned}
\frac{1}{p} \mid\{0 \leq k \leq & \left.p-1:\left|d_{x}\left(A_{\sigma^{k}(n p)}\right)-d_{x}(A)\right| \geq \varepsilon\right\} \mid \\
\leq & \frac{1}{p}\left|\left\{0 \leq k \leq n_{0}-1:\left|d_{x}\left(A_{\sigma^{k}(n p)}\right)-d_{x}(A)\right| \geq \varepsilon\right\}\right| \\
& +\frac{1}{p}\left|\left\{n_{0} \leq k \leq p-1:\left|d_{x}\left(A_{\sigma^{k}(n p)}\right)-d_{x}(A)\right| \geq \varepsilon\right\}\right| \\
= & \frac{H}{p}+\frac{1}{p}\left|\left\{n_{0} \leq k \leq p-1:\left|d_{x}\left(A_{\sigma^{k}(n p)}\right)-d_{x}(A)\right| \geq \varepsilon\right\}\right| \\
\leq & \frac{H}{p}+\frac{\delta}{2} .
\end{aligned}
$$

If $p$ is taken sufficiently large, we can write

$$
\frac{H}{p}+\frac{\delta}{2}<\delta .
$$

So, the sequence $\left\{A_{k}\right\}$ is Wijsman quasi-invariant statistically convergent to $A$.

\section{REFERENCES}

[1] Baronti, M. and Papini, P., Convergence of sequences of sets, in: Methods of Functional Analysis in Approximation Theory, ISNM 76, Birkhäuser, Basel, (1986), 133-155

[2] Beer, G., On convergence of closed sets in a metric space and distance functions, Bull. Aust. Math. Soc., 31 (1985), No. 3, 421-432

[3] Beer, G., Wijsman convergence: A survey, Set-Valued Analysis, 2 (1994), No. 1-2, 77-94

[4] Connor, J. S., The statistical and strong p-Cesàro convergence of sequences, Analysis, 8 (1988), No. 1-2, 47-64

[5] Fast, H., Sur la convergence statistique, Colloq. Math., 2 (1951), No. 3-4, 241-244

[6] Fridy, J. A., On statistical convergence, Analysis, 5 (1985), No. 4, 301-314

[7] Mursaleen, M., Invariant mean and some matrix transformations, Thamkang J. Math., 10 (1979), 183-188

[8] Mursaleen, M., Matrix transformation between some new sequence spaces, Houston J. Math., 9 (1983), No. 4, 505-509

[9] Mursaleen, M. and Edely, O. H. H., On the invariant mean and statistical convergence, Appl. Math. Lett., 22 (2009), No. 11, 1700-1704

[10] Nuray, F., Gök, H. and Ulusu, U., $\mathcal{I}_{\sigma}$-convergence, Math. Commun., 16 (2011), 531-538

[11] Nuray, F. and Rhoades, B. E., Statistical convergence of sequences of sets, Fasc. Math., 49 (2012), 87-99 
[12] Nuray, F., Quasi-invariant convergence in a normed space, Annals of the University of Craiova-Mathematics and Computer Science Series, 41 (2014), No. 1, 1-5

[13] Pancaroğlu, N. and Nuray, F., On invariant statistically convergence and lacunary invariant statistical convergence of sequences of sets, Progress in Applied Mathematics, 5 (2013), No. 2, 23-29

[14] Raimi, R. A., Invariant means and invariant matrix methods of summability, Duke Math. J., 30 (1963), No. 1, 81-94

[15] Šalát, T., On statistically convergent sequences of real numbers, Math. Slovaca, 30 (1980), No. 2, 139-150

[16] Savaş, E., Some sequence spaces involving invariant means, Indian J. Math., 31 (1989), 1-8

[17] Savaş, E., Strongly $\sigma$-convergent sequences, Bull. Calcutta Math., 81 (1989), 295-300

[18] Savaş, E. and Nuray, F., On $\sigma$-statistically convergence and lacunary $\sigma$-statistically convergence, Math. Slovaca, 43 (1993), No. 3, 309-315

[19] Schaefer, P., Infinite matrices and invariant means, Prog. Amer. Math. Soc., 36 (1972), No. 1, 104-110

[20] Ulusu, U. and Nuray, F., Lacunary invariant statistical convergence of double sequences of sets, Creat. Math. Inform., (accepted)

[21] Wijsman, R. A., Convergence of sequences of convex sets, cones and functions, Bull. Amer. Math. Soc., 70 (1964), No. 1, 186-188

[22] Wijsman, R. A., Convergence of sequences of convex sets, cones and functions II, Trans. Amer. Math. Soc., 123 (1966), No. 1, 32-45

DEPARTMENT OF MATHEMATICS

AFYON KOCATEPE UNIVERSITY

03200, AFYONKARAHISAR, TURKEY

E-mail address: egulle@aku.edu.tr

E-mail address: ulusu@aku.edu.tr 